Article

\title{
Evaluation of a Tai Chi Intervention to Promote Well-Being in Healthcare Staff: A Pilot Study
}

\author{
David Marshall ${ }^{1}$, Grainne Donohue ${ }^{2, *}$, Jean Morrissey ${ }^{3}$ and Brendan Power ${ }^{1}$ \\ 1 Ballyfermot Mental Health Centre, Dublin 10, Ireland; david.marshall@hse.ie (D.M.); \\ brendan.power1@hse.ie (B.P.) \\ 2 Trinity Centre for Practice and Healthcare Innovation, School of Nursing and Midwifery, Trinity College, \\ University of Dublin, College Green, Dublin 2, Ireland \\ 3 School of Nursing and Midwifery, Trinity College, University of Dublin, College Green, Dublin 2, Ireland; \\ morrisje@tcd.ie \\ * Correspondence: donohuga@tcd.ie
}

Received: 1 November 2017; Accepted: 16 January 2018; Published: 24 January 2018

\begin{abstract}
Whilst healthcare professions are already considered one of the most stressful occupations, workplaces are becoming busier, and the potential for workplace absenteeism and burnout has intensified. There is growing evidence that the mind-body practice of Tai Chi, which originated in China as a martial art, has value in treating or preventing many health-related problems, such as stress and anxiety, and that regular practice helps to significantly improve wellbeing, attention, focus, and resilience. This intervention provided 12 sessions of Tai Chi for a group of 12 multidisciplinary healthcare workers and was general wellbeing was measured pre- and post-intervention. Using a mixed methods research design, it was discovered that there were statistically significant gains in well-being during this timeframe with results indicating a strong case for further roll out of the program to a larger pool and more extensive study.
\end{abstract}

Keywords: Tai Chi; spirituality; healthcare workers; well-being

\section{Introduction}

There is growing evidence that the mind-body practice of Tai Chi has value in treating and preventing many health-related problems, such as stress and anxiety, and that regular practice helps to significantly improve well-being, attention, focus, and resilience (Lee and Ernst 2012; Sharma and Haider 2015; Wang et al. 2014). Working in healthcare means that staff are exposed to, and working with, people who present with complex, multi-layered, and challenging needs, which can be emotionally stressful for the worker (Corey and Corey 2011). In the context of helping, the need for healthcare staff to pay attention to their own physical, mental, and emotional well-being is paramount not only for the sake of themselves but also for their patients/clients, their work colleagues, and the organization (Morrissey 2015).

Against the background of increasing staff and resource cuts in the Irish health system (Health Service Executive 2015), health care staff members are working in challenging and uncertain times, required to face greater demands and higher expectations and accountability, sometimes with limited or inadequate support systems available. One major stress faced by many healthcare staff is the reality of having too much to do in too little time; as a result, workers may try to make every effort to provide the level of care that is necessary in an unrealistically short time, often resulting in stress for both the helper and the person being helped. Opportunities should therefore be provided to safeguard and promote the physical and mental health wellbeing for all employees, which in turn is likely to enhance the quality and efficacy of the health care services provided (Black 2012). 
Healthcare professions, such as nursing, are often defined as stressful occupations due to the nature of the job (Porter et al. 2000). Nurses are particularly exposed to a risk of stress-related problems, resulting in higher absenteeism, compassion fatigue, burnout, and staff turnover (Mark and Smith 2012; Smith and Gray 2000). In the work context, workers may over time begin to demonstrate signs of compassion fatigue (Figley 1995), resulting in boredom, negativity, disinterest in work, and/or a decreased ability to complete tasks. Failure to address or resolve such problems may result in more severe impairments for the worker, including feelings of helplessness and a sense of feeling stuck or trapped that may manifest in negative attitudes towards self, co-workers, work, and life itself (Skovholt and Trotter-Mathison 2011). It therefore seems reasonable that strategies are implemented to counteract the effects of stress, compassion fatigue, or burnout (Maslach 1982), one such strategy being the use of Tai Chi.

Tai Chi is an ancient form of exercise, a traditional mind-body exercise which originated in China as a martial art and a sublime means of self-defense and self-cultivation in the Taoist tradition, originating from Wudang Mountain and later spreading to Beijing and other major Chinese cities. Traditionally an authentic art form, it was only handed down through family lineages until the latter part of the twentieth century. It combines traditional Chinese culture, direct mind-to-mind transmission from master to student, and ceaseless practice and perseverance, and it has been practiced in China for hundreds of years with the goal of rebalancing the body's healing capacity by supporting a healthy balance of yin and yang (the Chinese concepts of opposing force within the body) and refining the flow of qi (a vital energy within the body), which ultimately leads to lasting peace of mind and deeper levels of consciousness (Lo et al. 1979). In Tai Chi practice, through the correct use of thoughts, feelings, and ideas, which are expressed naturally from within, without force, through a systematic set of circular movements or form, a path of feeling in the body that stimulates the body's meridians and acupuncture points is produced and cultivated. Through repeated gentle practice over time, the health of the mind and body greatly improves. Tai Chi has been reported to have both physical and psychological benefits, promoting balance control and flexibility and thereby benefitting musculoskeletal conditions, cardiovascular and respiratory functions, endocrine and immune functions, and mental health (Lee and Ernst 2012; Wang et al. 2010). The popularity of Tai Chi may be explained by its non-stress exercise cycle, the feeling of relaxation and well-being, and the potentiality of addressing multiple health needs physically, spiritually, and psychologically. The primary aim of this review is to evaluate the use of Tai Chi as a way to promote well-being in healthcare staff with the hypothesis that engagement in a 12-week Tai Chi course will increase well-being scores as measured through the Warwick-Edinburgh Mental Well-Being Scale (WEMWBS) (Tennant et al. 2007).

\section{Materials and Methods}

This mixed methods research study evaluated the effectiveness of Tai Chi in improving well-being of staff at a Dublin Primary Care and Mental Health Centre. Ethical approval was granted by the ethics committee of the School of Nursing and Midwifery, Trinity College, and a purposive sample was recruited from staff at the healthcare setting. Participants $(n=12)$ were invited to take part in the intervention and to complete an anonymous online questionnaire via Survey Monkey pre and post-intervention regarding their overall well-being using the Warwick-Edinburgh Mental Well-Being Scale (WEMWBS) (Tennant et al. 2007). They were also asked some open-ended questions about the perceived impact of the intervention, which will be discussed later in this section. These responses were initially coded, and these codes were subsequently analyzed and subject to a thematic analysis (Braun and Clarke 2006). Participants were recruited via staff email lists and once consenting, anonymously filled out questionnaires online via Survey Monkey pre and post-study. An initial class of 12 health professionals enrolled in the 12-week course, and the program was facilitated by a qualified Tai Chi Instructor. 


\subsection{Instruments Used}

WEMWBS (Tennant et al. 2007) is a 14-item scale of mental well-being covering subjective well-being and psychological functioning, in which all items are worded positively and address aspects of positive mental health. The minimum scale score is 14 and the maximum is 70 . Responses are made on a 5-point scale ranging from "none of the time" to "all of the time." Each item is worded positively and together they cover most, but not all, attributes of mental well-being including both hedonic and eudemonic perspectives. Participants were also asked a series of open-ended questions based on their motivation for participation, expectations (both collected at baseline) and experience of the intervention, including benefits and challenges (collected post-intervention). These responses were collected anonymously online through an invite whereby participants were directed to a link via Survey Monkey. The results of this survey were then imported into SPSS version 16.0 for analysis.

\subsection{Details of Intervention}

A 45 min class once a week was taught over 12 weeks focusing on the 8 so-called "active ingredients" of Tai Chi leading to increased wellbeing and overall health both in body and in mind. This particular type of Tai Chi intervention was chosen based on a review of previous studies, in particular the Harvard Medical School Guide to Tai Chi (Wayne 2013). The offer of a simplified Tai Chi program as outlined below included all key elements in a short and succinct formula so that participants could achieve maximum benefits without having to learn complex movements of forms as would be done traditionally. The $45 \mathrm{~min}$ session was comprised of $10 \mathrm{~min}$ of "Neigong" (sitting meditation to activate mindfulness, focused attention, and increased awareness of the body and mind) followed by $20 \mathrm{~min}$ of "Qigong" (standing meditation, which focuses on structural alignment and integration, intention using imagery and visualization, active and passive relaxation, natural breathing, and increased awareness of natures' rules of balance through dynamic movements) and moving meditation or "Tai Chi" for $15 \mathrm{~min}$ to increase strength and flexibility, embodying movement through form and function, natural breathing, and the connection of thoughts/attention, ideas/feelings, and actions/movements, simplified into repeated movements so that participants could easily remember what they did in the class and thus practice at home on their own. These sessions took place in the healthcare center where participants worked so as to encourage participation and to minimize drop out.

\section{Results}

The total study participants included 10 females and 2 males and all were aged between 28 and 56 years. Their working roles were mainly categorized as nursing $(45 \%)$ but also included Occupational Therapy, Psychology, Administration and Medical professionals, all working within mental health services.

The data obtained through the questionnaire were initially gathered online through Survey Monkey. This data was transferred to the Statistical Package for the Social Sciences (SPSS) program, version 16.0 for Windows, for statistical analysis. This analysis was carried out using the $t$-test (used for variables with normal distribution to reject a null hypothesis or not). A paired sample $t$-test was conducted to evaluate the impact of the intervention on healthcare workers scores on the WEMWBS scale (Tennant et al. 2007). There was a statistically significant increase in well-being scores from Time 1 $(\mathrm{M}=50.88, \mathrm{SD}=7.31)$ to Time $2(\mathrm{M}=55, \mathrm{SD}=5.55), \mathrm{t}(7)=2.4, p<0.05$ (two-tailed). The mean difference in WEMWBS scores was 4.13 with a $95 \%$ confidence interval ranging from 0.06 to 8.1 . The eta squared statistic (0.45) indicated a large effect size. The results indicate that well-being scores significantly improved with participants who took part in the 12-week Tai Chi program.

In order to further consider the impact of the intervention, an additional analysis was conducted on the open-ended questions asked of participants through the online questionnaire pre- to post-study using a thematic analysis (Braun and Clarke 2006). Coding was manually conducted by a researcher 
independent of the intervention and sample, and themes were developed on the basis of these codes. This analysis revealed the motivations of participants who signed up for the intervention, which included, but were not limited to, "the opportunity to engage in well-being practice in work," "curiosity," "interest in trying something new," and "creating a space for self-care in the workplace." Expectations of the intervention ranged from the hope of learning a new skill to reducing stress levels and finding a moment of calm in the day. One participant had no expectations for the intervention and another hoped it would allow them "to focus on the task at hand as [they] often struggle with switching off." In further analyzing some of these participant responses about their experience of the intervention, two striking themes became evident reflecting the personal and occupational impact and are detailed in the next sections.

\subsection{Personal: Stress Management}

All of the participants of this research without exception expressed positive benefits to taking part in the Tai Chi intervention. All expressed a sense of achieving calm through the exercises and a renewed perception of worries and ongoing stress, both personal and work-related:

Definitely, calmer and more relaxed overall. Thinking differently about stuff and if it's worth the worry.

I feel more relaxed and grounded at work, thoughts racing less, feeling more calm and content in myself

Energy levels are higher. Reduced stress levels. Sleep pattern improved (on night duty)

Moments to yourself where you clear your mind of distractions are hard to manufacture in the modern era and are wonderful oases of calm.

The intervention in itself was an activity enjoyed by participants as a break from the weekly routine:

I look forward to it every week and feel calmer and more grounded I feel that it shows me that my workplace values me more

I would say I feel more relaxed. I look forward to the class every week.

\subsection{Occupational: Creating a Positive Workplace}

Participants were also unanimous in their reflections on being part of this intervention and the positive impact that it has had on their work environment. It was seen as both a progressive and constructive investment in the work space and a creation of something unique:

It's so worthwhile. Our work can become very stressful at times and this is a great opportunity to unwind.

I definitely felt more positive about having that space in work and I think that filtered into how I felt in work, in a good way

Great sharing a protected space with colleagues

It certainly made work a more welcoming prospect on a Thursday.

This positive addition was particularly highlighted by participants as important for people working specifically within the caring profession:

I feel it was hugely beneficial for staff to practice during work hours and it helps to calm the body and mind. Working as care givers, we tend to spend all of our energy on others, neglecting ourselves, so I think we all need to be more mindful of our own self-care.

We need to take to support our own wellness and recovery to help us to support the wellness and recovery of service users. I also enjoyed the experience and have recommended it to friends. I believe 
that that Tai Chi is a way in which we can address individual and organization wellbeing and cultural change.

It is very important for us as mental health professionals to maintain our own stress, and the class allows us protective and supportive time.

\section{Discussion}

\subsection{Stress Management}

Healthcare staff report high levels of stress and burnout that are costly in terms of quality of care, personal wellness, and productivity. In one survey of 2500 nurses, approximately $75 \%$ of respondents reported working with some pain; nearly one in five also reported depressive symptoms (Letvak et al. 2012). Clearly, strategies designed to improve the health and wellbeing of healthcare staff are in the best interest of the staff themselves, the institutions where they work, and the patients they treat. This pilot study tested the effect of onsite Tai Chi classes to improve well-being and the reduction of personal stress was a key finding from participants. These results correlate with a systematic review (Wang et al. 2010), whereby Tai Chi has been shown to be positively associated with improvement in stress levels in healthy adults.

\subsection{Creating a Positive Workplace}

There is mounting evidence that work-based wellness offerings are cost-effective (Goetzel and Ozminkowski 2008) and may impact productivity (Goetzel et al. 2007). In a period of declining resources for many health care organizations, it is only prudent therefore to introduce and evaluate workplace wellness interventions with implications for employee wellbeing included in the design. Creating a "culture of health" has been advocated to promote health behavior change and improve the health of individuals (Shareck et al. 2013). It has been identified that this "culture of health" can be achieved through settings-based health promotion and intervention (World Health Organisation WHO). Workplaces are an integral setting for improving health (Black and Frost 2011), and workplace-health-based interventions have been shown to reduce the burden of illness to both employees and the employer. Furthermore, it is often assumed that mental health problems only develop outside of the workplace and are not the responsibility of the employer, but there is growing evidence that poor psychosocial working conditions, commonly referred to as "job stressors," can increase the risk for developing symptoms, including depression, anxiety, burnout, and distress (Harvey et al. 2017; Joyce et al. 2016). Offering wellbeing intervention to staff such as the Tai Chi practice described in this study goes some way in offering an effective way to address these ongoing concerns.

\section{Conclusions}

This study analyzed the experience of mental healthcare professionals taking part in a 12-week, once weekly Tai Chi intervention. The results show that participants demonstrated a statistically significant improvement in terms of well-being as measured by the WEMWBS (Tennant et al. 2007) and their open-ended responses to questions on the intervention demonstrated how this affected them both at a personal and occupational level.

Practices that prioritize the physical and mental wellbeing of caring professionals are still lacking. These findings highlight the potential opportunities related to the introduction of a spiritually based practice to a mental health care setting. Findings support the introduction of such an intervention as a positive wellness option in the workplace, thus encouraging replication with a larger sample.

Offering an innovative approach that aims to strengthen the ability of mental healthcare professionals to cope with increased demands through maintaining their own health and level of vitality is an attractive prospect. The strength of Tai Chi is its range of self-care options that meet the scheduling, physical ability level, and preference of varied groups of professionals. As discussed 
earlier, the creation of this shared space may build a sense of service-wide cohesiveness and offer benefits that extend beyond one's specific unit.

In terms of study limitations, as the nature of this study is a small-scale pilot study, the findings may not be generalized and are context-dependent in terms of being undertaken in a single country-Ireland-and within a single health service provider. In addition, a control group to compare scores was absent. In qualitative analysis, only one member of the research team conducted the analysis; it would be recommended that any further research would utilize another researcher for inter rater reliability in the coding process.

Author Contributions: David Marshall conceived of and performed the intervention; Grainne Donohue analyzed the data and edited the paper; Jean Morrissey contributed to the design of the study and editing of the paper; Brendan Power was involved in the initial stages of the research design. David Marshall is a Chief Instructor (also known as "Long Zhan") and a longtime student of the late G. M. Jeffrey J. Guiffre and the Yin Cheng family of Beijing (the late G. M. Wang Peisheng).

Conflicts of Interest: The authors declare no conflict of interest.

\section{References}

Black, Dame Carol. 2012. Why healthcare organizations must look after their staff. Nursing Management 19: 27-30. Black, Dame Carol, and David Frost. 2011. Health at Work; An Independent Review of Sickness Absence (Cm8205). London: The Stationary Office.

Braun, Virginia, and Victoria Clarke. 2006. Using thematic analysis in psychology. Qualitative Research in Psychology 3: 77-101.

Corey, Marianne Schneider, and Gerald Corey. 2011. Becoming a Helper, 6th ed. Belmont: Brookes Cole.

Figley, Charles R. 1995. Compassion fatigue: Toward a new understanding of the costs of caring. In Secondary Trauma Stress: Self-Care Issues for Clinicians, Researchers and Educators. Edited by B. H. Stamm. Lutherville: Sidran Press, pp. 3-28.

Goetzel, Ron Z., and Ronald J. Ozminkowski. 2008. The health and cost benefits of work site health-promotion programs. Annual Review of Public Health 29: 303-23.

Goetzel, Ron Z., David Shechter, Ronald J. Ozminkowski, Paula F. Marmet, Maryam J. Tabrizi, and Enid Chung Roemer. 2007. Promising practices in employer health and productivity management efforts: Findings from a benchmarking study. Journal of Occupational and Environmental Medicin 49: 111-30.

Harvey, Samuel B., Matthew Modini, Sadhbh Joyce, Josie S. Milligan-Saville, Leona Tan, Arnstein Mykletun, Richard A. Bryant, Helen Christensen, and Philip B. Mitchell. 2017. Can work make you mentally ill? A systematic meta-review of work-related risk factors for common mental health problems. Journal of Occupational Environmental Medicine 74: 301-10.

Health Service Executive. 2015. Corporate Plan for the Health Services 2015-2017. Available online: http: / / www.cuh.hse.ie/Careers/HSE-Corporate-Plan-2015-2017.pdf (accessed on 23 January 2018).

Joyce, Sadhbh, Matthew Modini, Helen Christensen, Arnstein Mykletun, Richard Bryant, Philip B. Mitchell, and Samuel B. Harvey. 2016. Workplace interventions for common mental disorders: A systematic meta-review. Psychological Medicine 46: 683-97. [CrossRef]

Lee, Myeong Soo, and Edzard Ernst. 2012. Systematic reviews of tai chi: An overview. British Journal Sports Medicine 46: 713-18.

Letvak, Susan A., Christopher Ruhm, and Sat N. Gupta. 2012. Nurses' presenteeism and its effect on self-reported quality of care and costs. American Journal of Nursing 112: 30-38.

Lo, Benjamin Pang Jeng, Martin Inn, Robert Amacker, and Susan Foe. 1979. The Essence of T'ai Chi Ch'uan: The Literary Tradition. Berkeley: North Atlantic, ISBN 0913028630.

Mark, G. G., and Andrew P. Smith. 2012. Occupational stress, job characteristics, coping and the mental health of nurse. British Journal of Health Psychology 17: 505-21.

Maslach, Christina. 1982. Burnout: The Cost of Caring. Englewood Cliffs: Prentice-Hall.

Morrissey, Jean. 2015. Self-Care: Professional and Personal Considerations. In Working with Self-Harm E Suicidal Behaviour. Edited by Louise Doyle, Brian Keogh and Jean Morrissey. London: Palgrave Macmillan, pp. 86-99.

Porter, Michael, Beth Alder, and Charles S. Abraham. 2000. Psychology and Sociology Applied to Medicine. London: Churchill Livingstone. 
Shareck, Martine, Katherine L. Frohlich, and Blake Poland. 2013. Reducing social inequities in health through settings-related interventions-A conceptual framework. Global Health Promotion 20: 39-52.

Sharma, Manoj, and Taj Haider. 2015. Tai chi as an alternative and complimentary therapy for anxiety: A systematic review. Journal of Evidence Based Complimentary Alternative Medicine 20: 143-53. [CrossRef] [PubMed]

Skovholt, Thomas M., and Michelle Trotter-Mathison. 2011. The Resilient Practitioner Burnout Prevention and Self-Care Strategies for Counselors, Therapists, Teachers and Health Professionals, 2nd ed. London: Routledge.

Smith, Pam, and Benjamin Gray. 2000. The Emotional Labour of Nursing: How Student and Qualified Nurses Learn to Care. London: South Bank University.

Tennant, Ruth, Louise Hiller, Ruth Fishwick, Stephen Platt, Stephen Joseph, Scott Weich, Jane Parkinson, Jenny Secker, and Sarah Stewart-Brown. 2007. The Warwick Edinburgh mental well-being scale (WEMWBS): Development and UK validation. Health and Quality of Life Outcomes 5: 63.

Wang, Chenchen, Raveendhara Bannuru, Judith Ramel, Bruce Kupelnick, Tammy Scott, and Christopher H. Schmid. 2010. Tai Chi on psychological well-being: Systematic review and meta-analysis. BMC Complementary and Alternative Medicine 10: 23.

Wang, Yanan, Lu Zheng, Tianhong Hu, and Quanquan Zheng. 2014. Stress, burnout and job satisfaction: Case of police force in China. Public Personnel Management 43: 325-39. [CrossRef]

Wayne, Peter M. 2013. The Harvard Medical School Guide to Tai Chi: 12 Weeks to a Healthy Body, Strong Heart, and Sharp Mind. Boston: Shambhala Publications.

World Health Organisation (WHO). 2014. World Health Statistics 2014. Geneva: WHO.

(C) 2018 by the authors. Licensee MDPI, Basel, Switzerland. This article is an open access article distributed under the terms and conditions of the Creative Commons Attribution (CC BY) license (http:/ / creativecommons.org/licenses/by/4.0/). 